\title{
Proton strange form factors and SAMPLE experiments
}

\author{
Soon-Tae Hong* \\ Department of Science Education, Ewha Womans University, Seoul 120-750 Korea
}

(Dated: July 18, 2003)

\begin{abstract}
We study the chiral models such as the Skyrmion and chiral bag to investigate the baryon strange form factors associated with the SAMPLE experiments.
\end{abstract}

\section{INTRODUCTION}

Recently, the SAMPLE Collaboration reported the experimental data of the proton strange magnetic form factor through parity violating electron scattering at a small momentum transfer $Q_{S}^{2}=0.1(\mathrm{GeV} / \mathrm{c})^{2}[1]$

$$
G_{M}^{s}\left(Q_{S}^{2}\right)=+0.14 \pm 0.29 \text { (stat) } \pm 0.31 \text { (sys) n.m.. }
$$

On the other hand, baryons were described by topological solitons [2, 3, 4, 5, 6] and the MIT bag model [7] was later unified with the Skyrmion model to yield the chiral bag model (CBM) [8], which then includes the pion cloud degrees of freedom and the chiral invariance consistently. Moreover, the soliton was exploited to yield superqualiton [9] in color flavor locking phase [10].

The QCD is the basic underlying theory of strong interaction, from which low energy hadron physics should be attainable. Moreover, for hadron structure calculations, the coupling constant $g$ is not a relevant expansion parameter of QCD. Long ago, 't Hooft noted that $1 / N_{c}$ could be regarded as expansion parameter of QCD [11] where $N_{c}$ is the number of colors and $g N_{c}^{2}$ is kept constant. The properties of large $N_{c}$ limit of the QCD can be satisfied by the meson sector of the nonlinear sigma model such as the Skyrmion model.

Now, in order to study the hadron physics phenomenology, we start with the SU(3) Skyrmion lagrangian of the form $[3]$

$$
L=\int \mathrm{d}^{3} x\left[-\frac{f_{\pi}^{2}}{4} \operatorname{tr}\left(l_{\mu} l^{\mu}\right)+\frac{1}{32 e^{2}} \operatorname{tr}\left[l_{\mu}, l_{\nu}\right]^{2}\right]+L_{W Z W}
$$

where $l_{\mu}=U^{\dagger} \partial_{\mu} U$ and $U \in \mathrm{SU}(3)$ is described by pseudoscalar meson fields $\pi_{a}(a=1,2, \ldots, 8)$ and the topological aspects can be included via the WZW action [3]. Assuming maximal symmetry, we introduce the hedgehog ansatz $U_{0}$ embedded in the $\mathrm{SU}(2)$ isospin subgroup of $\mathrm{SU}(3)$ to yield the topological charge

$$
Q=-\frac{1}{2 \pi} \chi_{E}(\theta-\sin \theta \cos \theta)=1
$$

where $\theta$ is the chiral angle and $\chi_{E}$ is the Euler characteristic being an inter two in the spherical bag surface.

\section{BRST SYMMETRIES IN SKYRMION}

In order to define the spin and isospin one can quantize, in the SU(2) Skyrmion for instance, the zero modes via $U_{0} \rightarrow A U_{0} A^{\dagger}$ and $A(t)=a^{0}+i \vec{a} \cdot \vec{\tau}$ with $a^{\mu}$ being the collective coordinates. One can then obtain the Lagrangian $L=-m_{0}+2 i_{1} \dot{a}^{\mu} \dot{a}^{\mu}$ where the static mass $m_{0}$ and the moment of inertia $i_{1}$ are calculable in the Skyrmion model. Introducing the canonical momenta $\pi^{\mu}$ one can obtain the canonical Hamiltonian $H=m_{0}+\frac{1}{8 i_{1}} \pi^{\mu} \pi^{\mu}$. Note that the second-class geometrical constraints

$$
\Omega_{1}=a^{\mu} a^{\mu}-1 \approx 0, \quad \Omega_{2}=a^{\mu} \pi^{\mu} \approx 0
$$

should be treated via the Dirac brackets [12]. However, in the Dirac quantization, one has difficulties in finding the canonically conjugate pair, which were later overcome [13] by introducing pair of auxiliary Stuckelberg fields $\theta$ and

*Electronic address: soonhong@ewha.ac.kr 
$\pi_{\theta}$ with $\left\{\theta, \pi_{\theta}\right\}=1$. In the Skyrmion the first-class constraints $\tilde{\Omega}_{1}=a^{\mu} a^{\mu}-1+2 \theta$ and $\tilde{\Omega}_{2}=a^{\mu} \pi^{\mu}-a^{\mu} a^{\mu} \pi_{\theta}$ were constructed [14] to satisfy the strongly involutive Lie algebra $\left\{\tilde{\Omega}_{1}, \tilde{\Omega}_{2}\right\}=0$. Similarly, the first-class Hamiltonian was formulated to yield the baryon mass spectrum

$$
m_{B}=m_{0}+\frac{1}{2 i_{1}}\left[J(J+1)+\frac{1}{4}\right]
$$

with the isospin quantum number $J$. Here note that an additional global shift is due to the Weyl ordering correction. Following the BRST quantization scheme [15] with (anti)ghost and their Lagrangian multiplier fields, we obtain the BRST symmetric Lagrangian [14],

$$
L_{e f f}=-m_{0}+\frac{2 i_{1} \dot{a}^{\mu} \dot{a}^{\mu}-\dot{\theta} \dot{b}}{1-2 \theta}-\frac{2 i_{1} \dot{\theta}^{2}}{(1-2 \theta)^{2}}-2 i_{1}(1-2 \theta)^{2}(b+2 \bar{c} c)^{2}+\dot{\bar{c}} \dot{c}
$$

invariant under the transformations, $\delta_{\lambda} a^{\mu}=\lambda a^{\mu} c, \delta_{\lambda} \theta=-\lambda(1-2 \theta) c, \delta_{\lambda} \bar{c}=-\lambda b$ and $\delta_{\lambda} c=\delta_{\lambda} b=0$. (For more details of the BRST quantization of the $\mathrm{SU}(2)$ and $\mathrm{SU}(3)$ Skyrmions, see [14] and [16], respectively.)

\section{BARYON STRANGE FORM FACTORS}

Next, we consider the CBM which is a hybrid of two different models: the MIT bag model at infinite bag radius on one hand and Skyrmion model at vanishing radius on the other hand. (The explicit CBM lagrangian is given in Ref. [6] for instance.) In the CBM the total topological charge $Q$ in Eq. (1.3) is now splitted into the meson and quark pieces to satisfy the Cheshire cat principle [17]. Moreover, the quark fractional charge is given by sum of integer one (from valence quarks) and the quark vacuum contribution, which is also rewritten in terms of the eta invariant [18].

In the collective quantization of the CBM, we explicitly obtain the proton magnetic moment $[19,20]$

$$
\mu_{p}=\frac{1}{90}\left(9 I_{1}+24 I_{2}+12 I_{3}+16 I_{4}-4 I_{5}\right)+\frac{2 I_{6}}{1125}\left(9 I_{1}+4 I_{2}-8 I_{3}\right)
$$

with the inertia parameters $I_{n}(n=1, \ldots, 6)$ calculable in the CBM. Similarly we construct the baryon octet magnetic moments to reproduce the Coleman-Glashow sum rules [20,21] such as $U$-spin symmetries, $\mu_{\Sigma^{+}}=\mu_{p}, \mu_{\Xi^{0}}=\mu_{n}$ and $\mu_{\Xi^{-}}=\mu_{\Sigma^{-}}$.

Now we define the Dirac and Pauli EM form factors via

$$
\left\langle p+q\left|\hat{V}^{\mu}\right| p\right\rangle=\bar{u}(p+q)\left[F_{1 B}\left(q^{2}\right) \gamma^{\mu}+\frac{i}{2 m_{B}} F_{2 B}\left(q^{2}\right) \sigma^{\mu \nu} q_{\nu}\right] u(p)
$$

where $q$ is momentum transfer and $\sigma^{\mu \nu}=\frac{i}{2}\left(\gamma^{\mu} \gamma^{\nu}-\gamma^{\nu} \gamma^{\mu}\right)$ and $m_{B}$ is baryon mass. The Sachs form factors are then given by $G_{M}=F_{1 B}+F_{2 B}$ and $G_{E}=F_{1 B}+\frac{q^{2}}{4 m_{B}^{2}} F_{2 B}$ so that, at zero momentum transfer, the Pauli strange form factor is identical to the Sachs strange form factor: $F_{2 B}^{s}(0)=G_{M}^{s}(0)$. In the SAMPLE experiment, they measured the neutral weak form factor

$$
G_{M}^{Z, p}=\left(\frac{1}{4}-\sin ^{2} \theta_{W}\right) G_{M}^{p}-\frac{1}{4} G_{M}^{n}-\frac{1}{4} G_{M}^{s}
$$

with $G_{M}^{p}$ and $G_{M}^{n}$ being the proton and neutron Sachs form factors, to predict the proton strange form factor (1.1) which is positive value contrary to the negative values from most of the model calculations except the predictions [19, 22] of the $\mathrm{SU}(3) \mathrm{CBM}$ and the recent predictions of the chiral quark soliton model [23] and the chiral perturbation theory $[24,25]$. (See [6] for more details.) In the CBM the proton strange form factor is given by [19]

$$
F_{2 N}^{s}(0)=\frac{1}{60}\left(21 I_{1}-4 I_{2}-2 I_{3}-4 I_{4}-2 I_{5}\right)+\frac{I_{6}}{2250}\left(-129 I_{1}+76 I_{2}-52 I_{3}\right)
$$

which, after some algebra with the other baryon octet strange form factors, yields the sum rule for the proton strange form factor in terms of the baryon octet magnetic moments only (for the other baryon sum rules see [26])

$$
F_{2 N}^{s}(0)=\mu_{p}-\mu_{\Xi^{-}}-\left(\mu_{p}+\mu_{n}\right)-\frac{1}{3}\left(\mu_{\Sigma^{+}}-\mu_{\Xi^{0}}\right)+\frac{4}{3}\left(\mu_{n}-\mu_{\Sigma^{-}}\right) .
$$

Explicitly calculating the inertia parameters $I_{n}$ numerically in (3.4), we predict the proton strange form factor, 0.30 n.m. and exploiting the experimental data for the baryon octet magnetic moments in (3.5) we obtain 0.32 n.m.. These predictions are comparable to the SAMPLE experimental data (1.1) and are shown in Table 1, together with those of the other baryon strange form factors. 
TABLE I: The baryon octet strange form factors in the CBM

\begin{tabular}{lrrrr}
\hline Input & $F_{2 N}^{s}(0)$ & $F_{2 \Lambda}^{s}(0)$ & $F_{2 \Xi}^{s}(0)$ & $F_{2 \Sigma}^{s}(0)$ \\
\hline $\mathrm{CBM}$ & 0.30 & 0.49 & 0.25 & -1.54 \\
Exp & 0.32 & 1.42 & 1.10 & -1.10 \\
\hline
\end{tabular}

\section{CONCLUSIONS}

In conclusion, we discussed the SAMPLE experiments in the topological solitons such as the Skymion and chiral models to predict baryon strange form factors. We also exploited the Dirac quantization associated with ghost field degrees of freedom to construct the BRST invariant chiral Lagrangian. In future, it will be interesting to consider in these chiral models anapole form factors discussed in the literatures [25].

This work is financially supported in part by the Korea Science and Engineering Foundation Grant (R01-200000015).

[1] R. Hasty et al., Science 290, 2117 (2000); R.D. McKeown, Phys. Lett. B219, 140 (1989).

[2] T.H.R. Skyrme, Proc. Roy. Soc. A260, 127 (1961).

[3] G.S. Adkins, C.R. Nappi and E. Witten, Nucl. Phys. B228, 552 (1983); E. Witten, Nucl. Phys. B223, 422 (1983); B223, 433 (1983).

[4] M. Rho, A.S. Goldhaber and G.E. Brown, Phys. Rev. Lett. 51, 747 (1983).

[5] S.T. Hong, Phys. Lett. B417, 211 (1998).

[6] S.T. Hong and Y.J. Park, Phys. Rep. 358, 143 (2002).

[7] A. Chodos, R.L. Jaffe, K. Johnson and C.B. Thorn, Phys. Rev. D10, 10 (1974).

[8] G.E. Brown and M. Rho, Phys. Lett. B82, 177 (1979).

[9] D.K. Hong, M. Rho and I. Zahed, Phys. Lett. B468, 261 (1999); D.K. Hong, S.T. Hong and Y.J. Park, Phys. Lett. B499, 125 (2001).

[10] K. Rajagopal and F. Wilczek, Handbook of QCD, ed. M. Shifman (World Scientific, 2001), and references therein.

[11] G. 't Hooft, Nucl. Phys. B72, 462 (1974).

[12] P.A.M. Dirac, Lectures in Quantum Mechanics (Yeshiva University, New York, 1964).

[13] I.A. Batalin and E.S. Fradkin, Phys. Lett. B180, 157 (1986); I.A. Batalin and I.V. Tyutin, Int. J. Mod. Phys. A6, 3255 (1991).

[14] S.T. Hong and Y.J. Park, Phys. Rev. D59, 114026 (1999).

[15] C. Becci, A. Rouet and R. Stora, Ann. Phys. 98, 287 (1976); I.V. Tyutin, Lebedev Preprint 39 (1975).

[16] S.T. Hong and Y.J. Park, Phys. Rev. D63, 054018 (2000).

[17] M.A. Nowak, M. Rho, I. Zahed, Chiral Nuclear Dynamics (World Scientific, Singapore, 1996), and references therein.

[18] M.F. Atiyah, V. Patodi and I. Singer, Math. Proc. Camb. Phil. Soc. 77 (1975) 43.

[19] S.T. Hong and B.Y. Park, Nucl. Phys. A561, 525 (1993).

[20] S.T. Hong and G.E. Brown, Nucl. Phys. A564, 491 (1993).

[21] S. Coleman and S.L. Glashow, Phys. Rev. Lett. 6, 423 (1961).

[22] S.T. Hong, B.Y. Park and D.P. Min, Phys. Lett. B414, 229 (1997).

[23] H.C. Kim, M. Praszalowicz, M.V. Polyakov and K. Goeke, Phys. Rev. D58, 114027 (1998).

[24] Ulf-G. Meissner, Nucl. Phys. A666-A667, 51 (2000);

[25] C.M. Maekawa and U. van Kolck, Phys. Lett. B478, 73 (2000); U. van Kolck et al., Phys. Lett. B488 (2000) 167.

[26] S.T. Hong, hep-ph/0111470. 